\title{
Pressure and perceived coerciveness in an assertive community treatment program: an exploratory study Eric Latimer ${ }^{1,2}$, Anne G Crocker ${ }^{1,2}$, Olivier Farmer ${ }^{3}$ and A Todd Jenkins*1,2
}

\author{
Address: ${ }^{1}$ Douglas Hospital Research Center, 6875 LaSalle Blvd, Montreal QC H4H 1R3, Canada, ${ }^{2}$ McGill University, 845 Sherbrooke St West, \\ Montreal QC H3A 2T5, Canada and ${ }^{3}$ Centre Hospitalier de L'Universite de Montreal, 3480 Rue St Urbain, Montreal QC H2W 1T8, Canada \\ * Corresponding author
}

from WPA Thematic Conference. Coercive Treatment in Psychiatry: A Comprehensive Review

Dresden, Germany. 6-8 June 2007

Published: 19 December 2007

BMC Psychiatry 2007, 7(Suppl I):P9 doi:I0.II86/I47I-244X-7-SI-P9

This abstract is available from: http://www.biomedcentral.com/I47I-244X/7/SI/P9

(c) 2007 Latimer et al; licensee BioMed Central Ltd.

\section{Background}

Limited evidence suggests that Assertive Community Treatment (ACT) teams may in some cases be unnecessarily coercive. The study aimed to adapt or develop measures of client-perceived coerciveness, and of actual pressure or coercion, in an ACT setting. It also aimed to examine the association between these two constructs, as well as their associations with client sociodemographic and clinical characteristics.

\section{Methods}

Uses of pressure or coercion in a high-fidelity ACT team in Montreal, Canada were observed over an eight-week period. Based on these observations, the MacArthur Admission Experience Interview and Survey were adapted to the ACT setting to measure coercion perceived by clients. A new measure of pressure actually applied to clients, based on client self-reports, was also developed. Both measures were administered to a sample of 38 consenting clients. Sociodemographic and clinical measures, symptoms and level of functioning were also measured and correlated with the coercion measures.

\section{Results}

Most clients report having been subject to relatively low levels of pressure by ACT team members, and perceived coerciveness also appears low. The two constructs, however, are not correlated. Clients who function at a lower level and those who are more symptomatic report being subject to more pressure; among these, only the former also perceive the team as more coercive.

\section{Conclusion}

Actual and perceived coerciveness appear to represent two different constructs. Their levels in a mature, high-fidelity ACT program were low but not negligible. Separate monitoring of pressure applied to clients, and of perceived coerciveness, may be needed. 